University of Wollongong

Research Online

Faculty of Engineering and Information

Faculty of Engineering and Information

Sciences - Papers: Part A

Sciences

$1-1-2013$

Combined adaptive lattice reduction-aided detection and antenna shuffling for DSTTD-OFDM systems

Ngoc Phuc Le

University of Wollongong, pnl750@uowmail.edu.au

Le Chung Tran

University of Wollongong, Ictran@uow.edu.au

Farzad Safaei

University of Wollongong, farzad@uow.edu.au

Follow this and additional works at: https://ro.uow.edu.au/eispapers

Part of the Engineering Commons, and the Science and Technology Studies Commons

Research Online is the open access institutional repository for the University of Wollongong. For further information contact the UOW Library: research-pubs@uow.edu.au 


\title{
Combined adaptive lattice reduction-aided detection and antenna shuffling for DSTTD-OFDM systems
}

\author{
Abstract \\ In this paper, we consider lattice reduction (LR) aided linear detection in a DSTTD-OFDM (double space- \\ time transmit diversity- orthogonal frequency division multiplexing) system with antenna shuffling. We \\ first derive an antenna shuffling criterion for the LR-aided DSTTD-OFDM system. Next, we propose a \\ combined reduced-feedback and adaptive LR algorithm by exploiting the correlation between OFDM \\ subcarriers in the frequency domain. The LR-aided DSTTD OFDM system with this algorithm requires low \\ computational effort for the LR operation and small feedback information. Simulation results show that a \\ significant improvement could be achieved in the proposed system compared to previous (non-LR-aided) \\ systems under spatially correlated channels. Also, the proposed complexity-reduced approach could \\ greatly lower the system complexity while exhibiting a slight performance loss.
}

\section{Keywords}

antenna, detection, aided, reduction, lattice, adaptive, combined, systems, ofdm, dsttd, shuffling

\section{Disciplines}

Engineering | Science and Technology Studies

\section{Publication Details}

N. Le, L. Chung. Tran \& F. Safaei, "Combined adaptive lattice reduction-aided detection and antenna shuffling for DSTTD-OFDM systems," in 14th IEEE International Workshop on Signal Processing Advances in Wireless Communications (SPAWC 2013), 2013, pp. 100-104. 


\title{
Combined Adaptive Lattice Reduction-Aided Detection and Antenna Shuffling for DSTTD-OFDM Systems
}

\author{
Ngoc Phuc Le, Le Chung Tran, and Farzad Safaei \\ School of Electrical, Computer and Telecommunications Engineering \\ The University of Wollongong \\ Northfields Avenue, NSW 2522, Australia \\ Emails: \{pnl750, lctran, farzad\}@uow.edu.au
}

\begin{abstract}
In this paper, we consider lattice reduction (LR) aided linear detection in a DSTTD-OFDM (double space-time transmit diversity- orthogonal frequency division multiplexing) system with antenna shuffling. We first derive an antenna shuffling criterion for the LR-aided DSTTD-OFDM system. Next, we propose a combined reduced-feedback and adaptive LR algorithm by exploiting the correlation between OFDM subcarriers in the frequency domain. The LR-aided DSTTDOFDM system with this algorithm requires low computational effort for the LR operation and small feedback information. Simulation results show that a significant improvement could be achieved in the proposed system compared to previous (non-LRaided) systems under spatially correlated channels. Also, the proposed complexity-reduced approach could greatly lower the system complexity while exhibiting a slight performance loss.
\end{abstract}

Keywords- DSTTD; MIMO; antenna shuffling; adaptive lattice reduction; MB-OFDM UWB.

\section{INTRODUCTION}

Double space-time transmit diversity (DSTTD) [1] is a hybrid combination of space-time block codes (STBC) (e.g. Alamouti code [2]) and spatial multiplexing. This coding scheme is very attractive as it could simultaneously provide spatial multiplexing gain and transmit diversity gain. In fact, the DSTTD scheme has been adopted in some wireless systems, such as WiMAX [3]. Due to the very high complexity of the optimal maximum-likelihood (ML) detector, especially when higher modulation schemes are used, many suboptimal but simple detectors, such as zero-forcing (ZF) or minimum mean-squared error (MMSE), are considered for DSTTD systems. Unfortunately, the DSTTD system with suboptimal detectors suffers from huge performance degradation, especially in spatially correlated channels [1]. To further improve the performance of DSTTD systems in the spatially correlated scenario, an antenna shuffling method which selects the appropriate pairs of antennas for each STBC component is employed [1], [4]. The investigation of a DSTTD-OFDM (orthogonal frequency division multiplexing) system with a proposed antenna shuffling criterion has been performed in [5]. However, the obtained performances with conventional linear detectors are still poor, compared to the optimal ML detector. It is clear that developing an efficient detection method for the DSTTD scheme is of importance for DSTTD-OFDM systems with or without antenna shuffling.
In [6], H. Yao and G. Wornell have shown that using lattice reduction (LR) technique could significantly enhance the performance of MIMO (multi-input multi-output) systems. Moreover, the existing results [7]-[9] show that LR-aided linear detectors could achieve the same diversity order as that collected by the optimal ML detector while requiring much lower complexity. Interestingly, the complexity of LR-aided detectors can be further reduced with an adaptive LR approach [10]. Although this adaptive LR concept is originally proposed in a single-carrier system under time-correlated fading channels, it could be extended to OFDM systems.

Motivated by these works, we propose a DSTTD-OFDM system with an alternative antenna shuffling criterion and adaptive LR-aided linear detection in this paper. We first derive an antenna shuffling criterion by minimizing the determinant of the error covariance matrix of LR-aided linear detectors. The advantage of this shuffling criterion is that the obtained shuffling pattern is calculated directly from estimated channel coefficients. Therefore, the proposed system requires no additional complexity regarding the shuffling process in comparison with systems without lattice reduction. Next, by exploiting the fact that the neighboring subcarriers within an OFDM symbol are correlated, we both extend the adaptive LR concept to our system to further reduce the complexity of the receiver and implement feedback reduction to lower feedback information. In particular, we develop a combined reducedfeedback and adaptive LR algorithm, based on which the proposed system could enjoy low complexity and small feedback overhead. It will be shown that the proposed system could offer much better error performance while requiring slightly increased complexity compared to previous systems.

Notation: A bold letter denotes a vector or a matrix, whereas an italic letter denotes a variable. $(.)^{*},(.)^{\mathrm{T}},(.)^{H},(.)^{-1}$, $\|\|,. E\{$.$\} , and \operatorname{det}($.$) denote complex conjugation, transpose,$ Hermitian transpose, inverse, vector norm, expectation, and determinant, respectively. $\mathbf{I}_{n}$ indicates the $n \times n$ identity matrix.

\section{LR-AIDED DSTTD-OFDM WITH ANTENNA SHUFFLING}

\section{A. System Model}

We consider a DSTTD-OFDM system with $n_{T}=4$ transmit antennas and $n_{R} \geq 2$ receive antennas as shown in Fig.1. At the transmitter, the input symbols are demultiplexed 


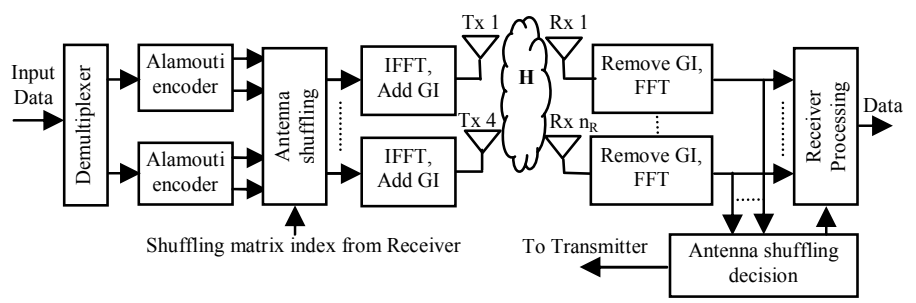

Fig. 1 Block diagram of a LR-aided DSTTD-OFDM with antenna shuffling

into two independent streams. Each data stream is then encoded using the Alamouti scheme [2]. When there is no antenna shuffling, the output sequences from the encoders are then fed into $N$-point IFFT blocks. Each IFFT output is added with a guard interval (GI) before being transmitted via its corresponding transmit antenna. At the receiver, the received signal at each antenna is fed into the FFT block after the GI is removed. Assuming that the fading coefficients remain constant during two consecutive OFDM symbol periods, the system model in the frequency domain corresponding to the $k^{\text {th }}, 1 \leq k \leq K$, subcarrier could be expressed as [1], [5]

$$
\mathbf{r}_{k}=\mathbf{H}_{k} \mathbf{s}_{k}+\mathbf{n}_{k}
$$

where $^{1} \quad \mathbf{s}_{k}=\left[\begin{array}{llll}s_{1} & s_{2} & s_{3} & s_{4}\end{array}\right]^{T}$,

$$
\begin{aligned}
\mathbf{H}_{k} & =\left[\begin{array}{cccc}
h_{1,1} & h_{1,2} & h_{1,3} & h_{1,4} \\
h_{1,2}^{*} & -h_{1,1}^{*} & h_{1,4}^{*} & -h_{1,3}^{*} \\
\vdots & \vdots & \vdots & \vdots \\
h_{n_{R}, 1} & h_{n_{R}, 2} & h_{n_{R}, 3} & h_{n_{R}, 4} \\
h_{n_{R}, 2}^{*} & -h_{n_{R}, 1}^{*} & h_{n_{R}, 4}^{*} & -h_{n_{R}, 3}^{*}
\end{array}\right], \\
\mathbf{r}_{k} & =\left[\begin{array}{lllll}
r_{1,1} & r_{1,2}^{*} & \ldots & r_{n_{R}, 1} & r_{n_{R}, 2}^{*}
\end{array}\right]^{T}, \\
\mathbf{n}_{k} & =\left[\begin{array}{lllll}
n_{1,1} & n_{1,2}^{*} & \ldots & n_{n_{R}, 1} & n_{n_{R}, 2}^{*}
\end{array}\right]^{T} .
\end{aligned}
$$

In the above equations, $\mathbf{s}_{\mathrm{k}}$ is the transmit symbol vector with $E\left\{\mathbf{s}_{k} \mathbf{s}_{k}^{H}\right\}=\mathbf{I}_{n_{T}}, h_{j, i}$ indicates the channel coefficient from the $i^{\text {th }}$ transmit antenna to the $j^{\text {th }}$ receive antenna, $r_{j, 1}$ and $r_{j, 2}$ denote the received signals on the $j^{\text {th }}$ receive antenna at the first and second periods, respectively. Also, $n_{j, 1}$ and $n_{j, 2}$ denote the noise on the $j^{\text {th }}$ receive antenna at the first and second periods, respectively. The noise is modeled as a Gaussian random variable with zero mean and variance $\sigma_{n}^{2}$. Note that the channel model $\mathbf{H}_{k}$ has the following property [1]

$$
\mathbf{H}_{k}^{H} \mathbf{H}_{k}=\left[\begin{array}{cc}
a_{k, 1} \mathbf{I}_{2} & \mathbf{B}_{k} \\
\mathbf{B}_{k}^{H} & a_{k, 2} \mathbf{I}_{2}
\end{array}\right],
$$

where $\mathbf{I}_{2}=\left[\begin{array}{ll}1 & 0 \\ 0 & 1\end{array}\right], \mathbf{B}_{k}=\left[\begin{array}{cc}b_{k, 1} & b_{k, 2} \\ -b_{k, 2}^{*} & b_{k, 1}^{*}\end{array}\right]$,

$$
\begin{aligned}
& a_{k, 1}=\sum_{j=1}^{n_{R}}\left(\left|h_{j, 1}\right|^{2}+\left|h_{j, 2}\right|^{2}\right), a_{k, 2}=\sum_{j=1}^{n_{R}}\left(\left|h_{j, 3}\right|^{2}+\left|h_{j, 4}\right|^{2}\right), \\
& b_{k, 1}=\sum_{j=1}^{n_{R}}\left(h_{j, 1}^{*} h_{j, 3}+h_{j, 2} h_{j, 4}^{*}\right), b_{k, 2}=\sum_{j=1}^{n_{R}}\left(h_{j, 1}^{*} h_{j, 4}-h_{j, 2} h_{j, 3}^{*}\right) .
\end{aligned}
$$

\footnotetext{
${ }^{1}$ The index $k$ associated with elements in Eq. (2)-(5) is omitted for simplicity.
}

\section{B. Lattice Reduction- aided Linear Detection}

In our system, a lattice reduction approach in a complex domain is adopted to obtain low complexity [8]. Thus, the system model in (1) could be written as [6], [8]

$$
\mathbf{r}_{k}=\mathbf{H}_{k} \mathbf{s}_{k}+\mathbf{n}_{k}=\mathbf{H}_{k} \mathbf{T}_{k} \mathbf{T}_{k}^{-1} \mathbf{s}_{k}+\mathbf{n}_{k}=\mathbf{G}_{k} \mathbf{z}_{k}+\mathbf{n}_{k},
$$

where $\mathbf{T}_{k}$ is an unimodular matrix which contains only Gaussian integer elements and $\left|\operatorname{det}\left(\mathbf{T}_{k}\right)\right|=1$. This matrix is obtained by using a complex LR algorithm that is based on the LLL (Lenstra-Lenstra-Lovasz) algorithm in [11]. Detailed description of the complex LR algorithm could be found in [8] and [9]. In this paper, we denote this algorithm as $\left[\mathbf{G}_{k}, \mathbf{T}_{k}\right]=\operatorname{CLLL}\left(\mathbf{H}_{k}, \mathbf{P}_{k}\right)$, initial setting $\mathbf{P}_{k}=\mathbf{I}_{n T}$ by default. Also, $\mathbf{z}_{k}=\mathbf{T}_{k}^{-1} \mathbf{s}_{k}$ is the transformed signal. $\mathbf{G}_{k}=\mathbf{H}_{k} \mathbf{T}_{k}$ is the reduced channel matrix that is much better conditioned, i.e. its columns are almost orthogonal, than the original matrix $\mathbf{H}_{k}$. The orthogonality of the reduced matrix could be measured using the orthogonality defect factor which is defined as [10]

$$
\vartheta\left(\mathbf{G}_{k}\right)=\frac{\prod_{u=1}^{n_{T}}\left\|\mathbf{g}_{u}\right\|^{2}}{\operatorname{det}\left(\mathbf{G}_{k}^{H} \mathbf{G}_{k}\right)},
$$

where $\mathbf{g}_{u}$ is the $u^{\text {th }}$ column of the reduced basis $\mathbf{G}_{k}$. Note that $\vartheta\left(\mathbf{G}_{k}\right) \geq 1$ with equality if and only if $\mathbf{G}_{k}$ is orthogonal.

The idea behind the LR-aided linear detection is applying a $\mathrm{ZF}$ or MMSE equalizer in the transformed domain to estimate $\mathbf{z}_{k}$, instead of $\mathbf{s}_{k}$. To this end, for the case of ZF detection, the equalized signal is computed as

$$
\widetilde{\mathbf{z}}_{k}=\mathbf{G}_{k}^{+} \mathbf{r}_{k}=\mathbf{z}_{k}+\left(\mathbf{G}_{k}^{H} \mathbf{G}_{k}\right)^{-1} \mathbf{G}_{k}^{H} \mathbf{n}_{k},
$$

where $\mathbf{G}_{k}^{+}=\left(\mathbf{G}_{k}^{H} \mathbf{G}_{k}\right)^{-1} \mathbf{G}_{k}^{H}$ is the Moore-Penrose pseudoinverse of matrix $\mathbf{G}_{k}$. The equalized signal is then quantized to produce $\hat{\mathbf{z}}_{k}$, and the final result is obtained as $\hat{\mathbf{s}}_{k}=\mathbf{T}_{k} \hat{\mathbf{z}}_{k}$. Note that as the columns in the matrix $\mathbf{G}_{k}$ are nearly orthogonal, the detection process carried out based on $\mathbf{G}_{k}$ will suffer from less noise enhancement than the conventional approach which is based on $\mathbf{H}_{k}$. In addition, a proper quantization is crucial for LR-aided detection. Detailed description of the quantization could be found in [7] and [8].

\section{Antenna Shuffling for LR-aided DSTTD-OFDM Systems}

When antenna shuffling is used, the receiver will determine the optimal shuffling pattern depending on the channel realization. The transmitter is then informed of the shuffling pattern index via a low-rate feedback channel. At the transmitter, a shuffling operation is implemented by multiplying the shuffling pattern with the encoded symbols. This shuffling operation is implemented for each subcarrier. Note that for the system with $n_{T}=4$ antennas, there are six shuffling patterns $\mathbf{W}$. The set of all $\mathbf{W}$ patterns is denoted as $\mathbf{S}(\mathbf{W})=\{[(1,2),(3,4)],[(1,2),(4,3)],[(1,3),(2,4)], \quad[(1,3),(4,2)]$, $[(1,4),(2,3)],[(1,4),(3,2)]\}[4]$. Therefore, 3-bit feedback on each subcarrier is required. Also, in the system with antenna shuffling, the elements of the equivalent channel model $\mathbf{H}_{k}$ are rearranged according to the chosen shuffling pattern $\mathbf{W}_{k}[1]$. 
With respect to a shuffling criterion operating associated with LR-aided detection, it is intuitive that the maximum mutual information criterion proposed in [4] could be applied. However, optimization criteria derived based on a specific receiver are preferred from a practical viewpoint. Therefore, we directly derive a shuffling criterion for our LR-aided system by minimizing the determinant of the error covariance matrix of LR-aided linear detectors. The shuffling criterion associated with the $k^{\text {th }}$ subcarrier is given as (see Appendix)

$$
\mathbf{W}_{k}=\arg \max _{\mathbf{W} \in \mathbf{S}(\mathbf{W})}\left(a_{k, 1} a_{k, 2}-\left(\left|b_{k, 1}\right|^{2}+\left|b_{k, 2}\right|^{2}\right)\right) .
$$

\section{Complexity Evaluation}

We now consider the complexity of the LR-aided DSTTDOFDM system in comparison with the non-LR counterpart. First, it is worth noting that the obtained shuffling pattern for (11) is computed directly from estimated channel coefficients, regardless of the LR operation. This means that, with respect to the antenna shuffling process, there is no additional complexity when lattice reduction is employed. On the other hand, it is shown in [6]-[9] that the deployment of LR-aided linear detection, especially when the CLLL algorithm is used, requires low additional complexity. For example, the complexity of the LR-aided ZF detector is about 1.7 times that of a ZF detector under uncorrelated channels [8]. In addition, the impact of channel correlation on the complexity of LRaided detectors is not serious and can be significantly reduced with a simple sorting step [9]. Consequently, the overall complexity of the proposed system is increased slightly compared to that of the system without lattice reduction.

\section{COMBINED FEEDBACK REDUCTION AND ADAPTIVE LATTICE REDUCTION FOR LR-AIDED DSTTD-OFDM SYSTEMS}

In Section II, we have proposed a LR-aided DSTTDOFDM system with antenna shuffling. In general, optimal performance could be achieved by performing antenna shuffling and LR-aided detection on a per-subcarrier basis. However, the complexity of the system with this approach might be high if the number of subcarriers in the OFDM system is large. By exploiting the fact that the neighboring subcarriers within an OFDM symbol are correlated, we could lower the complexity as well as the amount of feedback information with feedback reduction and adaptive LR.

1) Feedback reduction: Reduced feedback could be realized by combining subcarriers into a cluster and using only one proper shuffling pattern for all subcarriers in the cluster. Thus, with the cluster size of $L$, the number of feedback bits is reduced by $1 / L$. We propose the following criterion for choosing the shuffling pattern for the $m^{\text {th }}$ cluster, $1 \leq m \leq M$.

$$
\mathbf{W}_{m}=\arg \max _{\mathbf{W} \in \mathbf{S}(\mathbf{W})}\left\{\sum_{k=(m-1) L+1}^{m L}\left[a_{k, 1} a_{k, 2}-\left(\left|b_{k, 1}\right|^{2}+\left|b_{k, 2}\right|^{2}\right)\right]\right\} .
$$

Note that the choice of value $L$ depends on the coherence bandwidth of the channel.

2) Adaptive lattice reduction: Adaptive LR is originally proposed in a single-carrier system under time-correlated channels [10]. We now extend this concept to our DSTTD-
TABle I. A Proposed Complexity-Reduced Algorithm.

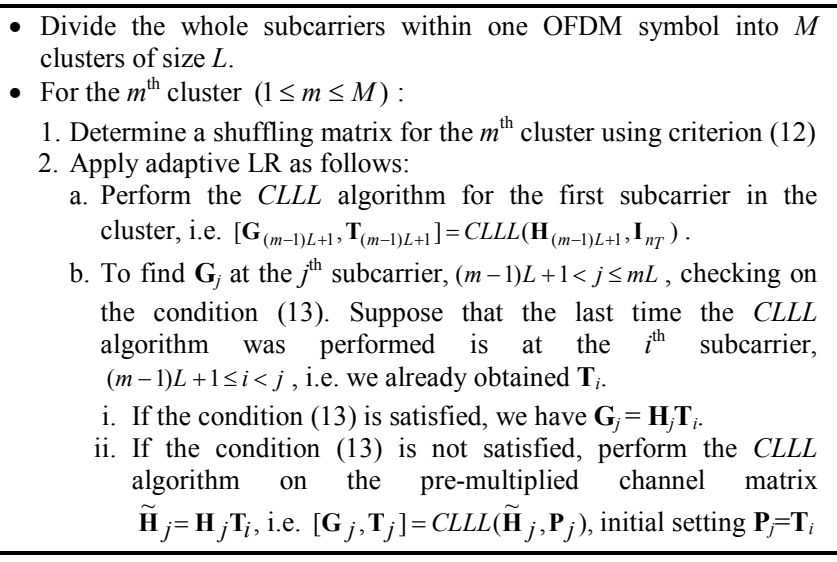

OFDM system. As the neighboring OFDM subcarriers are correlated, we note that the transformation matrix $\mathbf{T}_{\mathrm{k}}$ can be used to reduce the channel matrix of the adjacent subcarriers. The first approach is directly using $\mathbf{T}_{k}$ to reduce the original channel matrix of the adjacent subcarriers, i.e. without performing the CLLL algorithm. For the second approach, the original channel matrix of the adjacent subcarrier is first multiplied by $\mathbf{T}_{k}$. Then the CLLL algorithm is performed on the pre-multiplied matrix to obtain the reduced matrix. It is well-known that the complexity of LR mainly depends on the number of CLLL loops. Thus, performing the CLLL algorithm on the pre-multiplied channel matrix costs less complexity than that on the original matrix as the former is a better conditioned matrix. Suppose that the CLLL algorithm is performed at the $i^{\text {th }}$ subcarrier, i.e. $\left[\mathbf{G}_{i}, \mathbf{T}_{i}\right]=\operatorname{CLLL}\left(\mathbf{H}_{i}, \mathbf{P}_{i}\right)$, to determine which approach should be used to obtain $\mathbf{G}_{j}, j>i$, we measure the value $\vartheta\left(\mathbf{H}_{j} \mathbf{T}_{i}\right)$, and then check the condition

$$
\frac{\vartheta\left(\mathbf{H}_{j} \mathbf{T}_{i}\right)}{\vartheta\left(\mathbf{G}_{i}\right)} \leq \alpha
$$

where $\alpha \geq 1$ is chosen based on the desired performance, hardware complexity, and fading channel characteristics [10]. If (13) is satisfied, i.e. $\vartheta\left(\mathbf{H}_{j} \mathbf{T}_{i}\right)$ is good enough, we will use the first approach to obtain the reduced channel matrix.

A proposed algorithm that combines adaptive LR and feedback reduction is provided in Table I. In this algorithm, the choice of values $L$ as well as $\alpha$ is a matter of tradeoff between receiver complexity, feedback overhead, and error performance. Also, the adaptive LR operates on a cluster basis since the shuffling patterns for clusters are different in general.

\section{PERformance EVAluation}

In the simulations, we consider an uncoded $4 \times 2$ LR-aided DSTTD-OFDM system. The simulation parameters are chosen based on the WiMedia ${ }^{\circledR}$ Multiband-OFDM Ultrawideband standard [12] with a 4-QAM constellation and a FFT size of 128. We measure the system performance in terms of bit-error rate (BER) over the CM1 channel scenario defined in the IEEE 802.15.3a channel model [13]. In this model, the multipath gains are modeled as independent log-normally distributed random variables. The spatially correlated channel 


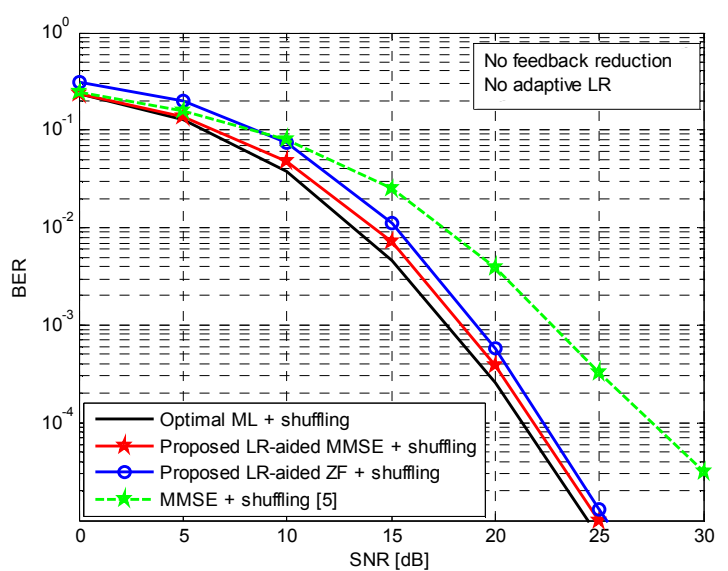

Fig. 2 Performance of the LR-aided DSTTD-OFDM system

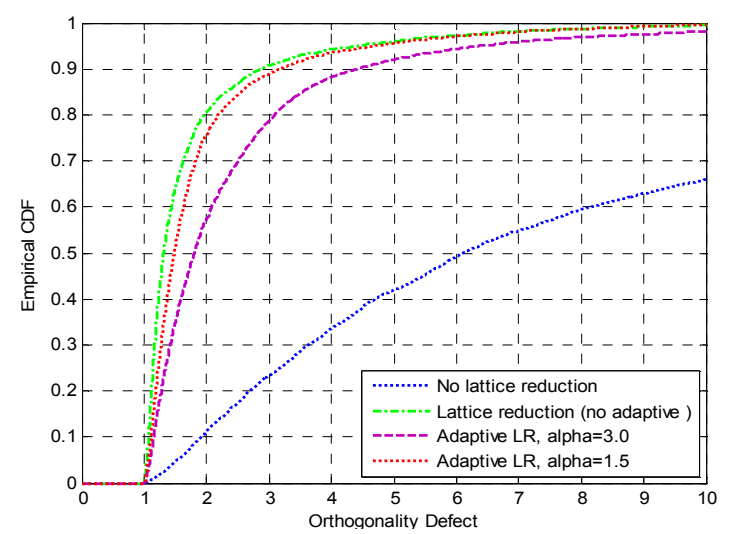

Fig. 3 Empirical CDF of the orthogonality defect of channel matrices

is modeled as $\mathbf{H}=\mathbf{R}_{R}^{1 / 2} \mathbf{H}_{i i d} \mathbf{R}_{T}^{1 / 2}$ [14], where $\mathbf{R}_{\mathrm{T}}$ and $\mathbf{R}_{\mathrm{R}}$ are the $n_{T} \times n_{T}$ correlation transmit matrix and the $n_{R} \times n_{R}$ receive correlation matrix, respectively. Also, $\mathbf{H}_{i i d}$ is a $n_{R} \times n_{T}$ channel matrix consisting of independent channel realizations obtained from the CM1 model for links between the transmit antennas and the receive antennas. We demonstrate the system performance in the correlated channel scenario with $\mathbf{R}_{\mathrm{T}}=$ toeplitz([ $\left.\left[\begin{array}{llll}1 & 0.9 & 0.81 & 0.729\end{array}\right]\right)$ and $\mathbf{R}_{\mathrm{R}}=\mathbf{I}_{2}$. We assume that perfect channel state information is available at the receiver.

\section{A. Peformance of LR-aided DSTTD-OFDM System}

It can be seen from Fig. 2 that the performance of the proposed system is relatively close to that of the DSTTDOFDM system with the optimal ML detector. Its BER curves are in parallel with that of the optimal ML detector, which exhibits its capability of collecting full diversity. Also, the proposed system outperforms the non-LR-aided counterpart proposed in [5]. For example, a SNR (signal-to-noise ratio) gain of about $5 \mathrm{~dB}$ at $\mathrm{BER}=10^{-4}$ could be achieved in the proposed system in comparison with the system in [5]. To have an insight on this improvement, we examine the orthogonality defect of the channel matrix. Fig. 3 shows the empirical CDF (cumulative distribution function) of the orthogonal defect of the channel matrices before and after performing LR. The CDFs plotted in the figure are average CDFs per subcarrier evaluated in a correlated scenario mentioned before. It is clear that the orthogonality defect of
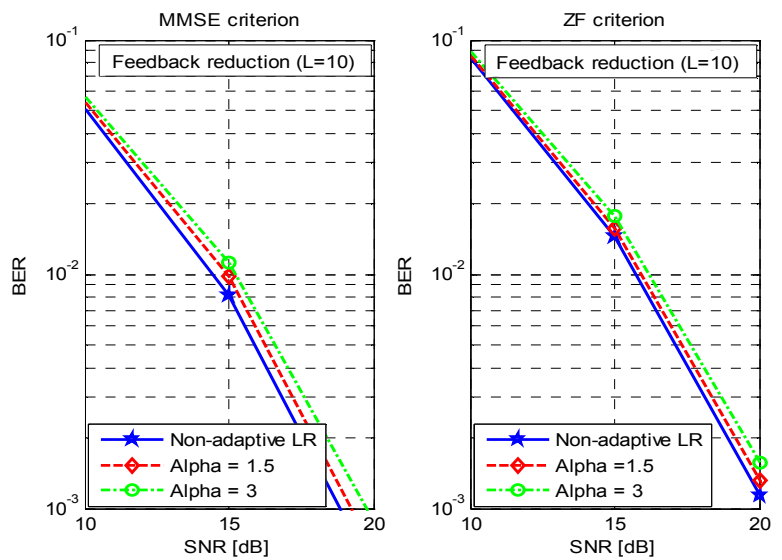

Fig. 4 Performance of the system with a reduced-complexity approach

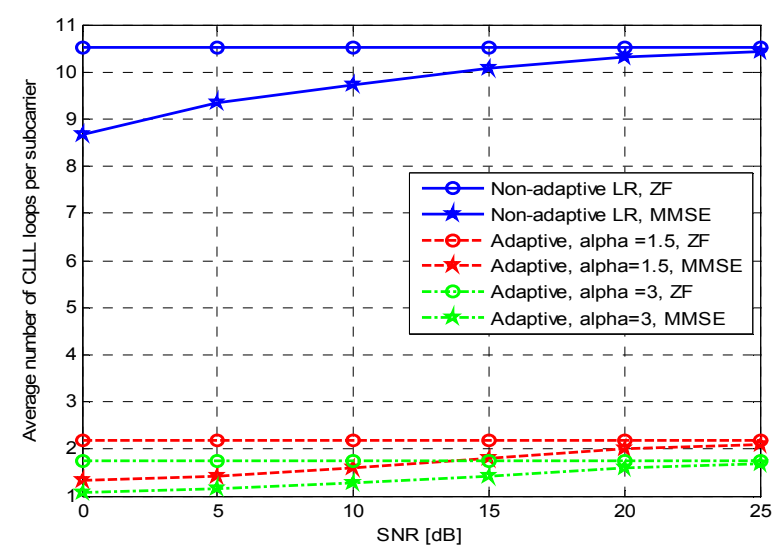

Fig. 5 Complexity comparison: adaptive LR vs. non-adaptive LR

the channel matrix is significantly reduced when using LR. Therefore, noise enhancement associated with LR-aided linear detection is much smaller than that in conventional linear detection. This explains the superiority in terms of error performance of the proposed system over the previous one.

\section{B. Performance of the LR-aided DSTTD-OFDM System with} Feedback Reduction and Adaptive Lattice Reduction

We evaluate the effectiveness of the proposed lowcomplexity approach by simultaneously considering BER performance and complexity aspects. Fig. 4 compares the BER performance of the adaptive LR system ( $\alpha=1.5$ and $\alpha=3$ ) to that of the non-adaptive LR counterpart for both ZF and MMSE criteria. Meanwhile, Fig. 5 shows their corresponding complexity in terms of a number of CLLL loops versus SNR. The feedback reduction of $L=10$ is used in all cases. Thus, only 30 feedback bits are required instead of 300 bits as in the system with no feedback reduction. It can be seen from Fig. 4 that there is some loss in performance when using adaptive LR compared to the case of non-adaptive LR. However, the performance gap is relatively small. Importantly, if we consider both the performance loss and the corresponding advantage in complexity, we will see the efficiency of the adaptive LR. For example, compared to non-adaptive LR, adaptive LR with $\alpha=1.5$ needs, on average, only $20 \%$ of CLLL loops per subcarrier while exhibiting about $0.5 \mathrm{~dB}$ performance loss. Note that the efficiency of the adaptive LR is also confirmed from the CDF curves shown in Fig. 3. 
TABLE II. AVERAge NumBer OF CLLL LOOPS PER SUBCARRIER.

\begin{tabular}{|l|c|c|c|c|c|}
\hline \multicolumn{1}{|c|}{ Correlation coefficient } & 0 & 0.2 & 0.4 & 0.6 & 0.8 \\
\hline Receiver & & & & & \\
\hline Non-adaptive ZF & 5.64 & 5.80 & 6.35 & 7.32 & 9.42 \\
\hline Non-adaptive MMSE & 5.47 & 5.64 & 6.15 & 7.08 & 9.11 \\
\hline Adaptive ZF, alpha=1.5 & 1.45 & 1.49 & 1.63 & 1.82 & 2.09 \\
\hline Adaptive MMSE, alpha=1.5 & 1.32 & 1.38 & 1.49 & 1.63 & 1.83 \\
\hline
\end{tabular}

\section{Impact of Spatial Correlation}

We now consider the effect of spatial correlation on the receiver complexity in terms of a number of $C L L L$ loops. Table III shows the average number of CLLL loops per subcarrier versus spatial correlation coefficients at $\mathrm{SNR}=15$ $\mathrm{dB}$ with $L=10$ for both adaptive LR and non-adaptive LR. It can be seen that the more correlated the channel is, the more CLLL loops are required. This makes sense as the channel becomes ill-conditioned under correlated scenarios, which in turn requires more loops in the CLLL algorithm for searching an almost orthogonal basis [9]. However, we observe that the adaptive LR is less sensitive to the spatial correlation. Moreover, the number of loops required in the adaptive LR system is small even when channels are highly correlated.

\section{CONCLUSIONS}

We have proposed an efficient LR-aided DSTTD-OFDM system with antenna shuffling and lattice reduction. By exploiting the correlation between subcarriers in the frequency domain, we have developed a complexity-reduced algorithm based on feedback reduction and adaptive lattice reduction. We have shown via analyses and simulations that the proposed system could offer much better BER performance while requiring low increased complexity compared to the previous systems. Also, the proposed system reveals some valuable properties, such as less sensitive to spatial correlation. These make the proposed system more attractive to the next generation of MIMO-OFDM based wireless communications.

\section{APPENDIX: DERIVATION OF (11)}

We first derive the antenna shuffling criterion for the LRaided DSTTD-OFDM system with LR-aided ZF detection. From (10), we could express the error covariance matrix as

$$
\mathbf{\Phi}_{e}=\mathrm{E}\left\{\left(\widetilde{\mathbf{z}}_{k}-\mathbf{z}_{k}\right)\left(\widetilde{\mathbf{z}}_{k}-\mathbf{z}_{k}\right)^{H}\right\}=\sigma_{n}^{2}\left(\mathbf{G}_{k}^{H} \mathbf{G}_{k}\right)^{-1} \text {. }
$$

We use a matrix optimization based on the determinant of a matrix [15]. Hence, the optimal pattern $\mathbf{W}_{k}$ is chosen so that the determinant of the error covariance matrix is minimized, i.e.

$$
\begin{gathered}
\mathbf{W}_{k}=\arg \min _{\mathbf{W} \in \mathbf{S}(\mathbf{W})} \operatorname{det}\left(\mathbf{\Phi}_{\mathbf{e}}(\mathbf{W})\right)=\arg \min _{\mathbf{W} \in \mathbf{S}(\mathbf{W})} \operatorname{det}\left(\sigma_{n}^{2}\left(\mathbf{G}_{k}^{H} \mathbf{G}_{k}\right)^{-1}\right) \\
=\arg \min _{\mathbf{W} \in \mathbf{S}(\mathbf{W})} \operatorname{det}\left(\left(\mathbf{G}_{k}^{H} \mathbf{G}_{k}\right)^{-1}\right)=\arg \max _{\mathbf{W} \in \mathbf{S}(\mathbf{W})} \operatorname{det}\left(\mathbf{G}_{k}^{H} \mathbf{G}_{k}\right) .
\end{gathered}
$$

On the other hand, as $\mathbf{G}_{k}=\mathbf{H}_{k} \mathbf{T}_{k}$ and $\left|\operatorname{det}\left(\mathbf{T}_{k}\right)\right|=1$, we have [16]

$$
\begin{aligned}
\operatorname{det}\left(\mathbf{G}_{k}^{H} \mathbf{G}_{k}\right) & =\operatorname{det}\left(\mathbf{T}_{k}^{H} \mathbf{H}_{k}^{H} \mathbf{H}_{k} \mathbf{T}_{k}\right) \\
& =\left|\operatorname{det}\left(\mathbf{T}_{k}\right)\right|^{2} \operatorname{det}\left(\mathbf{H}_{k}^{H} \mathbf{H}_{k}\right)=\operatorname{det}\left(\mathbf{H}_{k}^{H} \mathbf{H}_{k}\right) .
\end{aligned}
$$

Substitution of (16) into (15) yields

$$
\mathbf{W}_{k}=\arg \max _{\mathbf{W} \in \mathbf{S}(\mathbf{W})} \operatorname{det}\left(\mathbf{H}_{k}^{H} \mathbf{H}_{k}\right) \text {. }
$$

Perform similar calculations as in [4, Appendix], we obtain

$$
\mathbf{W}_{k}=\arg \max _{\mathbf{W} \in \mathbf{S}(\mathbf{W})}\left|a_{k, 1} a_{k, 2}-\left(\left|b_{k, 1}\right|^{2}+\left|b_{k, 2}\right|^{2}\right)\right| .
$$

In addition, as $\mathbf{H}_{k}{ }^{H} \mathbf{H}_{k}$ is a Hermitian positive semi-definite matrix, its eigenvalues $\left\{\lambda_{i}\right\}$ are real and non-negative [16]. Therefore, we have

$$
\lambda_{\text {min }}=\frac{\left(a_{k, 1}+a_{k, 2}\right)-\sqrt{\left(a_{k, 1}-a_{k, 2}\right)^{2}+4\left(\left|b_{k, 1}\right|^{2}+\left|b_{k, 2}\right|^{2}\right)}}{2} \geq 0 \text {. }
$$

Some simple manipulations on (19) yield

$$
a_{k, 1} a_{k, 2}-\left(\left|b_{k, 1}\right|^{2}+\left|b_{k, 2}\right|^{2}\right) \geq 0 \text {. }
$$

Combining (18) and (20) results in

$$
\mathbf{W}_{k}=\arg \max _{\mathbf{W} \in \mathbf{S}(\mathbf{W})}\left(a_{k, 1} a_{k, 2}-\left(\left|b_{k, 1}\right|^{2}+\left|b_{k, 2}\right|^{2}\right)\right) .
$$

It is straightforward to show that (21) is also the shuffling criterion for the case of MMSE detection due to the property of $\mathbf{H}_{\mathrm{k}}$ as shown in (6) and an equivalence between $\mathrm{ZF}$ and MMSE criteria with respect to the extended system model [7].

\section{REFERENCES}

[1] E. N. Onggosanusi, A. G. Dabak, and T. M. Schmidl, "High rate spacetime block coded scheme: performance and improvement in correlated fading channels," in Proc. IEEE WCNC 2002, pp. 194-199, March 2002.

[2] S. M. Alamouti, "A simple transmit diversity technique for wireless communications," IEEE J. Select. Areas Commun., vol. 16, no. 8, pp. 1451- 1458, Oct. 1998.

[3] IEEE Std 802.16e-2005, IEEE Standard for Local and Metropolitian Area Networks- Part 16: Air Interface for Fixed and Mobile Broadband Wireless Access Systems, Feb. 2006.

[4] H. Lee and E. J. Powers, "Low-complexity mutual information-based antenna grouping scheme for a D-STTD system," in Proc. IEEE Global Communications Conference, GLOBECOM 2006, pp. 1-5, Nov. 2006.

[5] L. Zhou and Y. Oishi, "Low complexity transceiver scheme for DSTTD OFDM system with antenna shuffling," in Proc. IEEE WCNC, pp. 171176, March 2008

[6] H. Yao and G. Wornell, "Lattice reduction aided detectors for MIMO communication systems," in Proc. IEEE Global Communications Conference, GLOBECOM 2002, pp. 17-21, Nov. 2002.

[7] D. Wübben, R. Böhnke, V. Kühn, and K. D. Kammeyer, "MMSE-based lattice detection for near ML detection of MIMO systems," in Proc. ITG Workshop Smart Antennas, pp. 106-113, March 2004.

[8] X. Ma and W. Zhang, "Performance analysis for MIMO systems with lattice-reduction aided linear equalization," IEEE Trans. Commun., vol. 56, no. 2, pp. 309-318, Feb. 2008.

[9] M. Sandell, A. Lillie, D. McNamara, V. Ponnampalam, and D. Milford, "Complexity study of lattice reduction for MIMO detection," in Proc. IEEE WCNC 2007, pp. 1089-1093, March 2007.

[10] H. Najafi, M. E. D. Jafari, and M. O. Damen, "On adaptive lattice reduction over correlated fading channels," IEEE Trans. Commun., vol. 59, no. 5, pp. 1224-1227, May. 2011.

[11] A. K. Lenstra, H. W. Lenstra, and L. Lovasz, "Factoring polynomials with rational coefficients," Math. Ann., vol. 261, pp. 515-534, 1982.

[12] A. Batra et al., "Multiband OFDM physical layer specification," WiMedia Alliance, Release 1.5, August 2009.

[13] J. Foerster et al., "Channel modeling sub-committee report final," IEEE P802.15-02/490r1-SG3a, Feb. 2003.

[14] T. Kaiser, F. Zheng, and E. Dimitrov, "An overview of ultrawideband systems with MIMO," Proc. IEEE, vol. 97, no. 2, Feb. 2009.

[15] W. W. Hager, Y. Liu, and T. F. Wong, "Optimization of generalized mean square error in signal processing and communication," ELSEVIER Linear Algebra and its Applications, pp. 815-834, 2006.

[16] G. H. Golub and C. F. Van Loan, Matrix Computation, $1^{\text {st }}$ Edition, Baltimore, MD: The Johns Hopkins University Press, 1983. 